\title{
Accuracy versus Complexity of MARG-based Filters for Remote Control Pointing Devices
}

\author{
Miguel Rasteiro $^{1}$, Hugo Costelha ${ }^{1,2}$, Luis Bento ${ }^{1,3}$ and Pedro Assuncao ${ }^{1,4}$ \\ ${ }^{1}$ Polytechnic Institute of Leiria / ESTG, ${ }^{2}$ INESC-TEC, ${ }^{3}$ ISR-UC, ${ }^{4}$ Instituto de Telecomunicacoes, Portugal
}

\begin{abstract}
Although most current pointing devices rely on relative rotation increments, absolute orientation allows for a more intuitive interaction. However, this is difficult to implement in lowenergy consumption devices since accurate fusion filters are computationally intensive. This work presents a comparative study of low-complexity filters and state-of-the-art orientation tracking systems, enabling to access complexity versus portability. A relevant set of different MARG units currently available on the market were studied under systematic tests and human subjective user analysis. Experimental results show that it is possible to obtain similar accuracy using low-complexity filters to the ones observed with state-of-the-art orientation tracking systems.
\end{abstract}

\section{INTRODUCTION}

In recent years there has been a huge evolution in user interaction with media content and an increasing convergence between interactive TV and multimedia personal devices such as, computers, tablets, and smartphones. However, content navigation devices for SmartTVs, Set-Top-Box or Media Centers did not follow this evolution. While the existing remote controls with air-mouse functionalities are becoming quite common, their use is not always intuitive or comfortable, leading to poor Quality of Experience (QoE). Usually, these devices are based on inertial movements, which are prone to drift over time, therefore requiring frequent position resetting.

In order to increase the QoE in interactive multimedia, one needs absolute navigation functionality. Remote control devices should be capable of autonomously compute its absolute orientation in a local coordinate system with good accuracy and low resetting frequency. Nowadays, the most common geometrical sensors layouts used for absolute orientation include 3 orthogonal axis of magnetometers, gyroscopes and accelerometers. Recently, Magnetic, Angular Rate, Gravity (MARG) units were made available encapsulating all three sensors types, into a single Integrated Circuit (IC) $4 \times 4 \times 1 \mathrm{~mm}^{3}$, often referred by the industry as $9 D$ sensor units. This integration allows reduced sensor noise, lower probability of axis misalignment, lower cost and lower size, which is particularly relevant in the design of remote control pointing devices.

Each sensor type can be characterized by its major application-specific source of disturbance: gyroscopes are prone to drift over time, accelerometers do not exclusively measure the gravity acceleration during regular operation of the remote control device and magnetometers are subjected to unpredictable disturbances in their usual application scenario, i.e. indoors. Magnetic disturbances, from sources such as building structure, nearby ferromagnetic materials and

This work is partially supported by European Union (COMPETE/QREN/FEDER) within project HERMES, number 34149 . appliances, can be difficult to compensate. This will induce error in the measurement of the earth magnetic field and bias the orientation estimation. An ellipsoid fitting calibration [1] can be applied to reduce the influence of the disturbance, but it can only cope with modelled disturbances.

Usually, some high computational filters are used to compute the orientation estimate and that may prevent the use of MARG units in wireless battery operated devices, due to power consumption, cost and latency. Therefore, determining if low complexity filters can achieve an acceptable level of accuracy for interactive multimedia applications, is a paramount task and it is addressed in this paper.

The experimental results were obtained with five different filters, two of which comprise an adaptive-gain feature and magnetic disturbances mitigation techniques. The proposed methods were implemented on embedded hardware and its performance evaluation is achieved via predefined trajectory path to assess the use of the device based on absolute orientation. The experiments were performed in an environment subject to high magnetic disturbance and high accelerations. All filters performance was comparatively analyzed along with state-of-the-art Commercial Off-The-Shelf (COTS) MARG units/filters. Also an assessment study for the preferred navigation functionality was performed, using a test application and three different air-mice. The overall results provide useful insight for developing user interface devices with increased QoE and reduced power requirements.

\section{MARG-BASED FILTERS}

The filters used to process MARG data combine the best attributes of each sensor type to reduce the overall error. There are two major approaches: Stochastic Filters (Kalman Filters (KF) [2] and Complementary Filters (CF) [3].

In the current study, a low-complexity KF and two different approaches of CF with two different implementations each, were tested. All implementations were done with quaternions due to their compact representation of orientation, lowcomplexity normalization and the fact that they do not suffer from representation singularities.

The KF is based on the work developed by Comotti et al. [2]. The CFs approaches were based on the work described by Madgwick et al. [4] and Mahony et al. [5]. In addition, some improvements were made to these CFs following the method proposed by Tian et al. [2]. The first CF tested is here denoted as Original Madgwick Filter (OMdF), and the improved version denoted as Adaptive-Gain Madgwick Filter (AGMdF). In this paper the filter proposed by Mahony et al. was implemented using quaternions, while the originally version used rotation matrices, therefore this filter is here denoted as Adapted Mahony Filter (AMhF), and the improved version as AdaptiveGain Mahony Filter (AGMhF). 


\section{Methodology}

The experiments were performed using an industrial robotic arm that could retrieve the MARG unit orientation at $15 \mathrm{~Hz}$ with a resolution of $0.01^{\circ}$ and a pose repeatability of $0.06 \mathrm{~mm}$, establishing the ground-truth used to calculate the performance of the implemented filters and state-of-the-art COTS MARG units/filters in a dynamic environment. A MPU9150 sensor from Invensense was used as the source of all raw data used in five tested filters. The state-of-the-art COTS MARG sensors used has reference were the following: the PNI SENTRAL M\&M Blue Module, which implements a patented method based on Extended KF (EKF), with continuous hard/soft-iron disturbance calibration and magnetic anomalies compensation; and a XSENS MTi-30 device with a proprietary XSens KF.

A survey was conducted to access the QoE by a human acting as an end user of tree different air-mouse implementations, henceforward designated by air-mouse 1, 2 and 3. The Movea company provides high performance solutions for TV remote control, designated as MoveaTV, in this paper the results of such system is presented as air-mouse 1, the MoveaTV solution used in the test doesn't use magnetometer data. The air-mouse 2 implements a relative navigation, with tilt compensation provided by the orientation information based on gyroscope, accelerometer and excluding magnetometer data. Air-mouse 3 provides absolute navigation based on the orientation of the device, directly converting Roll Pitch Yaw (RPY) angles into coordinates on the screen, based on all MARG sensors data.

The survey test involved 60 people, were, for each user, the following task was evaluated: navigate in a window and click on five buttons with different levels of difficulty according to button size. The survey enabled the user to grade in a 1-5 scale (being 5 the best grade), each air-mouse in terms of its accuracy, velocity and intuitiveness experienced.

\section{EXPERIMENTAL RESULTS}

The implemented filters have the capability to run with noncalibrated magnetometer data or even not using magnetometer data at all. The calibration of the magnetometer, using an ellipsoid fit method, improve the results, especially if it is calibrated in the environment where the MARG unit is to be used. The use of the magnetometer, in the tested environment, worsens the results for the heading angle error, but eliminates the drift over time and improves the general quality of results, i.e. the variance is reduced.

As can be seen in Figure 1, the adaptive-gain versions of CFs produce the best performance among the five tested filters. The results were obtained with the sensors mounted on the gripper off the industrial robot. Twelve run tests, each with 30 to 60 seconds, were executed over three different trajectories with ascending complexity in rotations.

Table I quantifies the computational burden of each filter, where the number of elementary operations was counted for an unoptimized $\mathrm{C}$ implementation of the filters. The KF is the most computationally intensive filter making it unsuitable for implementations on low-end (8-bit) microcontrollers.

Table II presents the results of the survey conducted through the use of a representative set of human users. The survey results reveal that the absolute positioning achieves the best results in almost all metrics used, having only a modest grade on the velocity parameter. Therefore the survey results evidence the user preference for the navigation with absolute orientation, the users found the Mouse 3 the most intuitive and accurate.

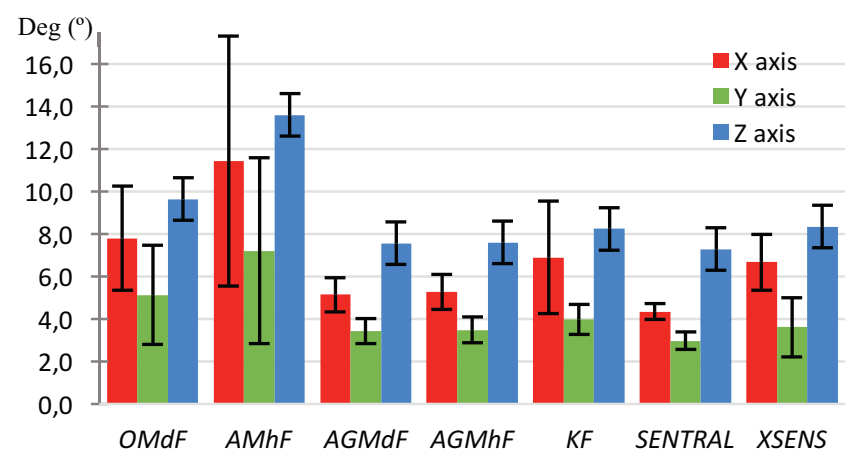

Fig. 1. Root Mean Square Error (RMSE) and its Standard Deviation (SD) for the implemented filters and reference sensors.

TABLE I - Number of operations per iteration for the implemented filters.

\begin{tabular}{lccccc} 
Filter & \#Multiplic. & \#Sums & \#Subtract & \#Divisions & \#Sq. roots \\
\hline AMdF & 121 & 46 & 31 & 10 & 4 \\
OMdF & 183 & 64 & 40 & 14 & 5 \\
AGMhF & 220 & 89 & 68 & 10 & 4 \\
AGMdF & 337 & 118 & 94 & 14 & 6 \\
KF & 625 & 255 & 115 & 24 & 6 \\
\hline
\end{tabular}

TABLE II - Survey results.

\begin{tabular}{cccc} 
Test & Accuracy & Velocity & Intuitiveness \\
\hline Mouse1 & 3,7 & 4,0 & 3,5 \\
Mouse2 & 3,6 & 4,4 & 3,8 \\
Mouse3 & 4,3 & 3,5 & 4,3 \\
\hline
\end{tabular}

\section{CONCLUSION}

This paper demonstrates that good accuracy results can be obtained using low-complexity complementary filters even in environments with high magnetic disturbances and undesirable accelerations. Absolute orientation seems to be the next step in multimedia interface devices as users tend to feel more comfortable using it. The results presented in this paper are useful to develop low-cost remote control pointing devices for future interactive media content and consumer equipment.

\section{REFERENCES}

[1] V. Renaudin, M. Afzal and G. Lachapelle, "New method for magnetometer based orientation estimation," in Position Location and Navigation Symposium (PLANS), 2010 IEEE/ION, May 2010, pp. 348-356.

[2] D. Comotti, M. Emidoro, M. Galizzi and A.Vitali, "Development of a Wireless Low-Power Multi-Sensor Network for Motion Tracking Applications," BSN IEEE Int. Conf., 2013.

[3] Ya Tian, H. Wei and J. Tan, "An Adaptive-Gain Complementary Filter for Real-Time Human Motion Tracking With MARG Sensors in Free-Living Environments," IEEE Trans. Neural Syst. Rehabil. Eng., vol.21, nº. 2, pp. 254-264, Mar. 2013.

[4] S. Madgwick, A. Harrison, and R. Vaidyanathan, "Estimation of IMU and MARG orientation using a gradient descent algorithm," Proc. IEEE Int. Conf. Rehabil. Robot., 2011, vol. 51, pp. 1-7.

[5] R. Mahony, T. Hamel, and J. M. Pflimlin, "Nonlinear complementary filters on the special orthogonal group," Automatic Control, IEEE Transactions on, 53(5):1203-1218, June 2008. 